\title{
Evidence of the Triangular Lattice of Crystallized Electrons from Time Resolved Luminescence
}

\author{
I. V. Kukushkin, ${ }^{1,2}$ Vladimir I. Fal'ko, ${ }^{1,2}$ R. J. Haug, ${ }^{1}$ K. von Klitzing, ${ }^{1}$ K. Eberl,${ }^{1}$ and K. Tötemayer ${ }^{1}$ \\ ${ }^{1}$ Max-Planck-Institut für Festkörperforschung, Heisenbergstrasse 1, 70569 Stuttgart, Germany \\ ${ }^{2}$ Institute of Solid State Physics, Russian Academy of Sciences, Chernogolovka, 142432, Russia
}

(Received 4 August 1993)

\begin{abstract}
We show that the recombination kinetics of two-dimensional electrons with acceptor bound holes is a sensitive probe of the local spatial structure of the electronic system. Using the time resolved magnetoluminescence, we extract the regime of the electron Wigner solid and establish its local configuration consistent with the triangular lattice model. Up to the melting point, the amplitude of the thermal vibrations of the electron crystal is derived from the temperature dependence of the recombination kinetics.
\end{abstract}

PACS numbers: $73.40 . \mathrm{Hm}, 73.40 . \mathrm{Kp}, 78.20 . \mathrm{Ls}$

The ground state of the two-dimensional (2D) electron system in the ultraquantum limit is formed by the interplay between electron-electron interaction and disorder. In a magnetic field both of them result in a frozen insulating state of the electronic system with different internal structures. In ideally pure systems, the formation of the Wigner solid state is expected [1], whereas the disorder hinders crystallization and gives rise to a state with no well-defined (even local) order. In high quality semiconductor structures, the importance of interaction has been indicated experimentally by the observation of the series of incompressible fractional states [2] which is terminated in high magnetic fields by an insulating phase, as it was observed in different experiments [3-9].

Several manifestations of the magnetically frozen state competing with the incompressible fractional liquid were also observed in magnetoluminescence experiments in which the recombination process involves the $2 \mathrm{D}$ electron and an acceptor bound photoexcited hole $h_{A}$ [8]. It was established that the entrance of the electron system into the insulating phase is accompanied by the appearance of an additional luminescence line [8] and a drastic reduction of the integral intensity $[8,10]$. The phase diagram built up from these optical studies [10] is similar to those obtained by other methods $[3-5,7,9]$. The threshold increase of the additional line intensity due to the electric field depinning of the frozen phase [8] indicates that this line is related to the recombination of localized electrons. In these experiments, the hole $h_{A}$ is strongly localized (within the acceptor-related Bohr radius), and its recombination efficiency is determined by the overlap of its wave function with that of a $2 \mathrm{D}$ electron: if electrons are localized and their states (in 2D plane) are squeezed inside the magnetic length, $\lambda_{H}$, this overlap diminishes for most of the holes and tends to zero under an increase of the magnetic field. This means that a quantity, such as the hole recombination rate, potentially possesses information about the short-range configuration of electrons in the magnetically frozen state [11].

The goal of the present work is to test the local spa- tial structure of the insulating phase of $2 \mathrm{D}$ electrons at high magnetic fields by means of the time resolved magnetoluminescence technique. The time integrated photoluminescence spectroscopy itself can hardly distinguish between different configurations (ordered or disordered) of frozen electrons in our system [12]. Nevertheless, the study of recombination kinetics at long time delays allows us to identify the short-range configuration of neighboring localized electrons as being consistent only with an ideal (equilateral) triangular structure with a very small portion of deformed cells. Moreover, the temperature dependence of the recombination tail is also consistent with the Debye-Waller-type factor expected for the magnetically frozen Wigner crystal correlated on a length which is much longer than the lattice constant.

We studied low-density $\left(n_{e}=6.1 \times 10^{10} \mathrm{~cm}^{-2}\right)$, highmobility $\left(3 \times 10^{6} \mathrm{~cm}^{2} / \mathrm{V} \mathrm{sec}\right) \mathrm{GaAs} / \mathrm{AlGaAs}$ single heterojunctions with a $\delta$-doped monolayer of Be acceptors $\left(n_{A}=1.3 \times 10^{9} \mathrm{~cm}^{-2}\right)$ located in the wide $(1 \mu \mathrm{m}) \mathrm{GaAs}$ buffer layer at a distance $30 \mathrm{~nm}$ from the interface [13]. For comparison, we also investigated a low-mobility $\left(10^{5}\right.$ $\mathrm{cm}^{2} / \mathrm{V} \mathrm{sec}$ ) single heterojunction with the same electron density and similar architecture (different only by the spacer width). For photoexcitation, we used pulses from a tunable Ti-sapphire laser (wavelength $\sim 800 \mathrm{~nm}$ ) with a variable duration from $20 \mathrm{~ns}$ to $10 \mu \mathrm{s}$, peak power of $10^{-5}-10^{-4} \mathrm{~W} / \mathrm{cm}^{2}$, and frequency of $10^{7}-10^{3} \mathrm{~Hz}$. The luminescence spectra were detected by a gated photon counting system. The samples were mounted in the mixing chamber of a ${ }^{3} \mathrm{He} /{ }^{4} \mathrm{He}$ dilution refrigerator. We control the temperature of $2 \mathrm{D}$ electrons and the time dependence of their density $n_{e}$ by monitoring the optical analog of the Shubnikov-de Haas oscillations [10]. For a laser wavelength of $\approx 800 \mathrm{~nm}$ at the above mentioned power level, $n_{e}$ does not change in time with an accuracy better than $3 \%$ after the pulse (contrary to the case of laser wavelength $488 \mathrm{~nm}[10])$. The time dependence of the magnetoluminescence intensity $I(t)$ measured at different magnetic fields (providing filling factors $\nu \leq 1$ ) is shown in Fig. 1. At $\nu=1$, the recombination kinet- 


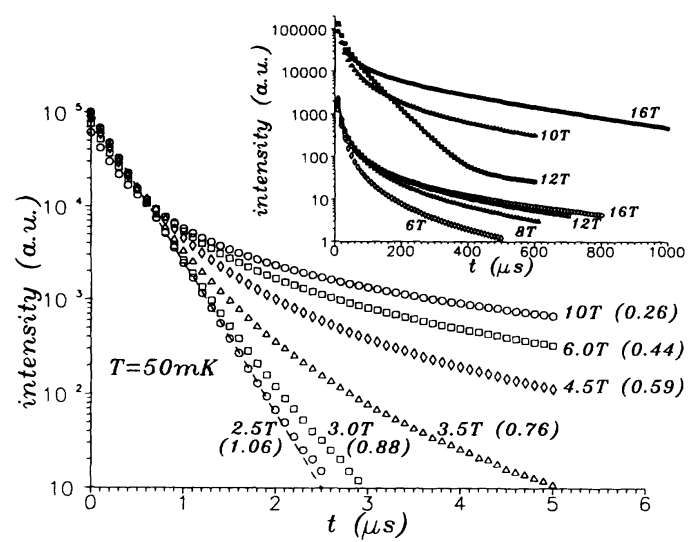

FIG. 1. Kinetics of luminescence measured for low-mobility sample in different magnetic fields. Inset shows high magnetic field kinetics measured up to $1000 \mu$ s time delay for low-mobility (open symbols) and high-mobility (closed symbols) samples. For clarity, the data for high-mobility samples are shifted.

ics can be clearly described by a single time constant. As the filling factor decreases below unity, the kinetics $I(t)$ deviate from the single-rate behavior and the slow recombination tail starts to dominate at $\nu \ll 1$, which is demonstrated in the inset to Fig. 1.

Before analyzing this slow recombination tail in detail, we discuss first the correspondence between the internal structure of the localized state and the observed magnetoluminescence kinetics. Since the density of holes in our system is several orders of magnitude less than the $2 \mathrm{D}$ electron density, the time dependence of the radiation process is governed by a single-hole recombination rate $\tau^{-1}$ which has the form [11] $\tau^{-1}=\sigma_{0} \rho\left(\mathbf{r}_{0}\right)$. In the latter expression, the value $\sigma_{0}$ includes all the magnetic field independent parameters, such as the optical transition strength in GaAs and the electron-hole overlap in the $z$ direction. Below it will be referred to as a phenomenological constant. On the other hand, the electron-hole overlap in the plane is controlled by the value $\rho\left(\mathbf{r}_{0}\right)=\left\langle 0\left|\hat{\psi}^{\dagger}\left(\mathbf{r}_{0}\right) \hat{\psi}\left(\mathbf{r}_{0}\right)\right| 0\right\rangle$ of the ground state $2 \mathrm{D}$ electron density matrix [14] related to the planar coordinate $\mathbf{r}_{0}$ of a hole position, which provides us with the relation between the hole recombination rate and the local internal structure of the electron system.

Since at $\nu=1$ the electron ground state possesses a homogeneous density $\rho(\mathbf{r})=n_{e}$ [15], all hole positions $\mathbf{r}_{0}$ are equivalent and the recombination kinetics absolutely agrees with the single exponent evolution of the luminescence intensity, $I(t)=I_{0} \exp \left(-t / t_{0}\right)$, with the rate $t_{0}^{-1}=\sigma_{0} n_{e}$. This simple single-rate description of the recombination process is immediately violated if the system leaves the homogeneous ground state. The holes $h_{A}$, randomly placed in the $\delta$-doped monolayer, have a distinguishably different lifetime because they test the locally modulated 2D electron density. At low filling factors (i.e., high magnetic fields), this modulation takes a drastic form if the electronic state is frozen. To imagine this, let us consider a Maki-Zotos type of state [16], $|0\rangle=N^{-1 / 2} \operatorname{det}\left\|\psi_{i}\left(\mathbf{r}_{\alpha}\right)\right\|$, prepared of electrons localized inside the magnetic length $\lambda_{H}$ near its own center $\mathbf{R}_{i}$, $\left|\psi_{i}(\mathbf{r})\right|^{2}=\left(2 \pi \lambda_{H}^{2}\right)^{-1} \exp \left[-\left(\mathbf{r}-\mathbf{R}_{i}\right)^{2} / 2 \lambda_{H}^{2}\right]$. The modulation $\rho(\mathbf{r})$ in such a system is exponential, and the temporal evolution of the intensity $I(t)$ follows some kind of a recombination "firefront" propagation through the distribution function $P(r)$ of distances $r$ between a hole and the closest electron localization center $\mathbf{R}_{i}$,

$I(t)=n_{h} \int P(r) d r \frac{\sigma_{0} e^{-r^{2} / 2 \lambda_{H}^{2}}}{2 \pi \lambda_{H}^{2}} \exp \left(-\frac{t \sigma_{0}}{2 \pi \lambda_{H}^{2}} e^{-r^{2} / 2 \lambda_{H}^{2}}\right)$.

The characteristic evolution of $I(t)$ can be illustrated by the example of the Poisson distribution of localization centers $\mathbf{R}_{i}, P(r)=2 \pi n_{e} r \exp \left(-n_{e} \pi r^{2}\right)$. In this case, we find that after the first holes placed just near their closest electrons are gone $\left(t \sim \nu t_{0}\right)$, the recombination takes the power law form $I(t)=I_{0}\left(2 \pi \lambda_{H}^{2} / \sigma_{0} t\right)=I_{0}\left(\nu t_{0} / t\right)^{1+\nu}$ which covers the entire interval $t>\nu t_{0}$. Although the distribution function $P(r)$ in realistic systems is affected by the electron-electron correlations and does not exactly coincide with a Poissonic one, the above power law is specific for any (even regular) configuration of localization centers as an intermediate regime. Indeed, at $r<n_{e}^{-1 / 2}$, $P(r) \approx 2 \pi n_{e} r$ [see inset to Fig. 2(a)]; hence for a wide time interval $\nu t_{0}<t<\nu t_{0} \exp (1 / \nu)$ the dependence

$$
I(t)=I_{0}\left(\nu t_{0} / t\right)^{1+\delta}
$$

(with $\delta \rightarrow 0$ at $\nu \rightarrow 0$ and $\delta \equiv 0$ in a crystal) seems to be a universal feature of the bound hole recombination in the electron system localized at high magnetic fields [11], independently of the details of its specific internal structure.

The result of Eq. (1) suggests an appropriate representation of the measured recombination kinetics in terms of a new dynamical variable: the instant value of the effective recombination time $\tau_{\text {eff }}(t)$ which is determined as $\tau_{\text {eff }}=-I(t) / \dot{I}(t)=-[d \ln I(t) / d t]^{-1}$. In coordinates $\tau_{\text {eff }}$ vs $t$, the kinetics described by a single exponent and by a power law will be represented by constant and linear dependences, respectively. For the power law decay in Eq. $(1), \tau_{\text {eff }}(t)=t /(1+\delta)$, which agrees with what is found in the experimental time dependence of $I(t)$. In Fig. 2(a) the data from Fig. 1 are replotted in the scale $\tau_{\text {eff }}$. One sees that, at fillings $\nu<1, \tau_{\text {eff }}$ starts to increase linearly with $t$ (at $t>t_{0}$ ) indicating the existence of localized electrons. Figures 2(a) and 2(b) show $\tau_{\text {eff }}$ for the low(b) and high- (c) mobility samples for longer times and smaller filling factors. It is visible that $\tau_{\text {eff }}(t)$ approaches $t$ for filling factors $\nu \ll 1$.

The latter regime is quite pronounced both in the most disordered (b) and in the purest (c) samples we studied, though the number of recombination events which form the long-delay tail are different at high magnetic fields. In our experiments, we found that in spite of extremely 

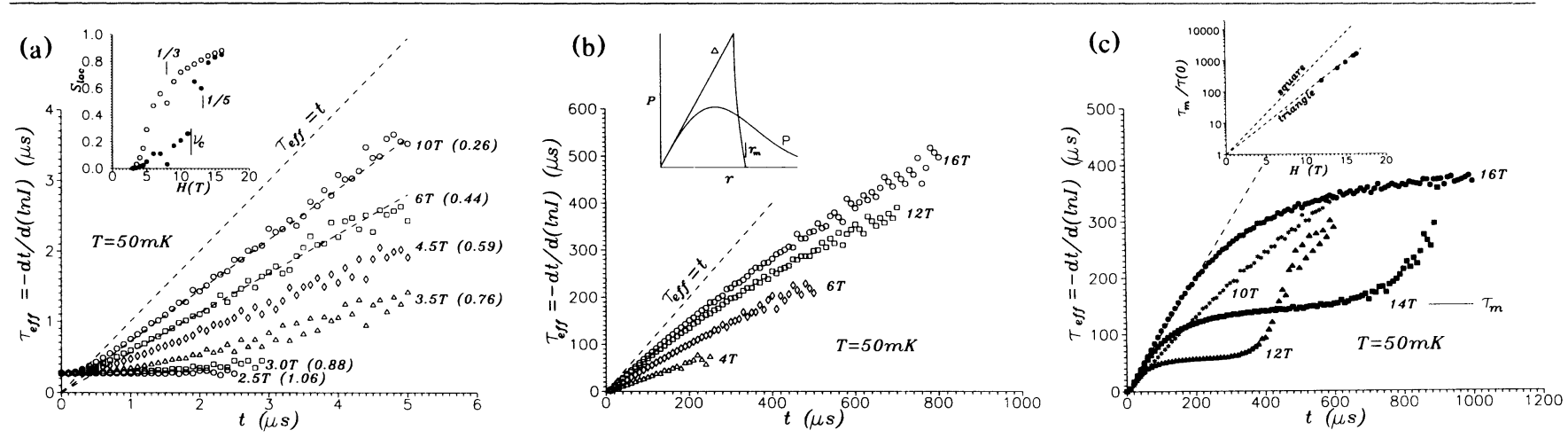

FIG. 2. Dependences of the instant recombination time $\tau_{\text {eff }}$ on time delay $t$ measured at different magnetic fields for low(a),(b) and high-mobility (c) samples. Insets show (a) magnetic field dependence of the portion of localized electrons $S_{\text {loc }}$, measured for low- (open symbols) and high-mobility (closed symbols) samples; (b) the distribution function $P(r)$ of distances between a hole and the closest electron for different configurations of electrons $(\mathrm{P}$, the Poisson distribution; $\triangle$, triangular lattice); (c) comparison of the measured magnetic field dependence of $\tau_{m}$ with that calculated for triangular (1) and square (2) lattices.

slow recombination at high fields, the total luminescence intensity integrated over time and energy is insensitive to the magnetic field. This means that the electron-hole recombination has $100 \%$ radiative efficiency, and thus, the integral intensity of the slow recombination tail which reflects the portion of localized electrons $S_{\text {loc }}$ in the 2D system can be measured in absolute units. In the lowmobility sample, $S_{\text {loc }}$ continuously grows starting from $\nu=1$ and tends to $100 \%$ as $\nu \rightarrow 0$ [see inset to Fig. $2(a)$. In high-mobility structures, the portion of localized electrons is much less, but abruptly increases at a critical filling factor $\nu_{c}$. This jump can be assigned to the phase transition of electrons from a liquid into the frozen state which is absent in the dirty sample. The phase diagram which can be reconstructed from the jump position is similar to previous accurate determinations $[3-5,7-10]$, and the following analysis is supposed to identify the structure of this frozen phase.

At the critical $\nu_{c}$ a pronounced part (about $50 \%$ ) of intensity is redistributed from the fast recombination channel to the slow recombination tail and this redistributed intensity shows a different behavior as compared to the long tail observed in the disordered sample [Figs. 2(c) and 2(b)]. This difference is most pronounced for delay times $t \geq t_{0} \exp (1 / \nu)$ when the recombination involves electrons and holes separated by the mean interparticle distance $l \sim n_{e}^{-1 / 2}$ and tests that part of the distribution function $P(r)$ which is dominantly affected by correlations in the local configuration of the closest frozen electrons. That is, in systems where the effect of disorder is stronger than that of the Coulomb interaction, the long tail in $P(r)$ spreads to lengths longer than $l$, and, although the effective recombination time $\tau_{\text {eff }}$ deviates from the linear intermediate regime, it does not saturate at any fixed value. On the contrary, the ordering of electrons into the Wigner lattice obviously demands the existence of a terminating point $r_{m}$ in the distribution function $P(r)$, as shown in the inset to Fig. 2(b).
This point corresponds to the distance from the lattice sites to the center of the unit cell, and has its minimal value (for a fixed density $n_{e}$ ) for the triangular lattice, $r_{m}^{\Delta}=(2 / 3 \sqrt{3})^{1 / 2} n_{e}^{-1 / 2}$. [For instance, the square lattice gives $r_{m}^{\diamond}=\left(2 n_{e}\right)^{-1 / 2}$; a strongly deformed glasslike structure possesses even longer spacings.]

After the recombination firefront reaches the terminating point $r_{m}$, the effective time $\tau_{\text {eff }}$ saturates at the lifetime value of a hole $h_{A}$ placed close to the unit cell center. Therefore, the saturation of the instant recombination time observed in the best samples [see Fig. 2(c)] means a single exponent recombination process and can be used for determining $r_{m}$. One can see from Fig. 2(c) and Fig. 3 that the plateau $\tau_{\text {eff }}(t) \approx \tau_{m}$ appears only when the filling factor and the temperature are below the critical values, $\nu<\nu_{c}$ and $T<T_{c}\left(\nu_{c}\right.$ and $T_{c}$ coincide with the values observed in transport [3-5] as well as in the luminescence measurements $[9,10])$.

The existence of the plateau in $\tau_{\text {eff }}$ has to be interpreted as the multiple repetition of a specific configuration of closest electrons. This configuration can be identified from the value of the normalized saturation time, $\tau_{m} / \tau_{\text {eff }}(t \rightarrow 0)=e^{r_{m}^{2} / 2 \lambda_{H}^{2}}=e^{\gamma / \nu}$. The structure factor $\gamma=\pi n_{e} r_{m}^{2}$ which enters into the exponent of this equation fits only to the triangular lattice for our results [see inset to Fig. 2(c)]. It cannot be assigned to other structures (for example, a square), since the reliability of such a procedure is guaranteed by the strong exponential dependence $e^{\gamma / \nu}$ and the fact that the ideal triangular lattice provides us with the absolute minimum of the value of $r_{m}$, as compared to any other frozen electron configuration with a fixed density. If the system contained a considerable portion of other ("deformed") configurations of frozen electrons which give rise to longer $r_{m}$, the effective recombination time $\tau_{\text {eff }}$ would not saturate at the value corresponding to the ideal triangle. Therefore, the number of defected cells is relatively small. They show up at the end of the single-exponential tail 


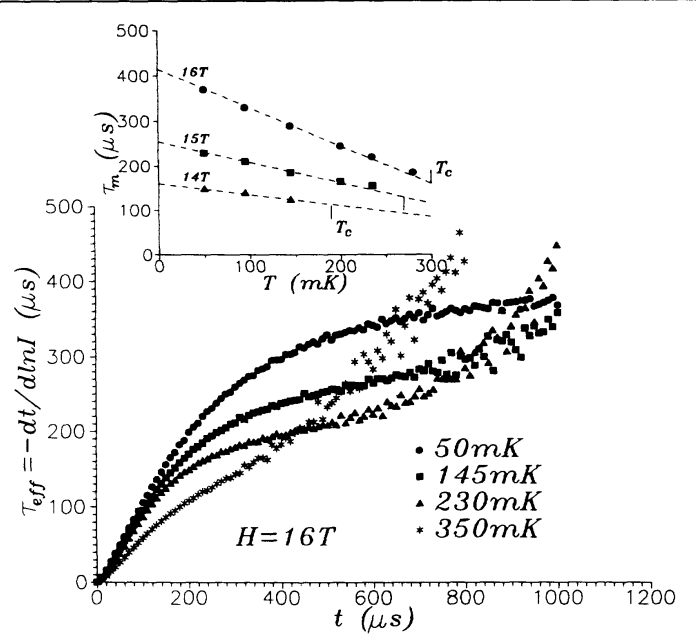

FIG. 3. Temperature variations of the dependence $\tau_{\mathrm{eff}}(t)$, measured for the high-mobility sample at $H=16 \mathrm{~T}$. Inset shows the temperature dependences of the $\tau_{m}$, measured at different magnetic fields.

$e^{-t / \tau_{m}}$ as a further increase of $\tau_{\text {eff }}$. The width $\Delta t$ of the plateau gives an estimate for the portion of defected cells, $I(\Delta t) / I\left(\tau_{m}\right) \sim e^{-\Delta t / \tau_{m}}$. It does not exceed $1 \%$ in our case [Fig. 2(c)]. All these facts force us to exclude a glasslike structure of the system, since in a glass the latter quantity should be close to unity.

The observed small portion of defected cells is consistent with the number of point defects (vacancies, interstitials, and dislocations [17]) expected from the number of charged acceptors and, most probably, indicates the softness of a 2D quantum crystal with a respect to share deformations. Charged acceptors together with a small amount of residual disorder pin the crystal and slightly deform it, which produces a finite correlation length $L$ of its long-range orientational ordering. From the portion of defected cells we can roughly estimate the correlation length to $L \geq n_{A}^{-1 / 2} \sim 300 \mathrm{~nm}$ which is 1 order of magnitude longer than the lattice constant. So the estimated correlation length of the crystal is also consistent with the temperature dependence of the saturation time, as discussed below.

Thermal vibrations (in high fields, magnetophonons with $k^{3 / 2}$ dispersion [18]) smear the electron density distribution and stimulate recombination in the long tail (Fig. 3). The temperature effect can be calculated [11] for holes placed in the center of a unit cell by renormalizing the density matrix value $\rho\left(\mathbf{r}_{m}\right)$ at this point. In the harmonic approximation, $\rho\left(\mathbf{r}_{m}\right)$ is proportional to $\exp \left(-r_{m}^{2} /\left[2 \lambda_{H}^{2}+\left\langle u^{2}\right\rangle_{T}\right]\right)$, where $\left\langle u^{2}\right\rangle_{T}$ is the mean square displacement of electron's magnetic oscillator center with respect to the regular site position. This produces the Debye-Waller-type factor in the recombination time $\tau_{m}$,

$$
\frac{\tau_{m}(T)}{\tau_{m}(0)} \approx \exp \left\{-\frac{\gamma \pi}{\nu^{2}} n_{e}\left\langle u^{2}\right\rangle_{T}\right\}
$$

where $\gamma=\pi n_{e} r_{m}^{2}$ is determined by the unit cell struc- ture $\left(\gamma_{\Delta}=2 \pi / 3 \sqrt{3}\right.$ and $\left.\gamma_{\diamond}=\pi / 2\right)$. Therefore, the amplitude of lattice vibrations is a measurable quantity in our experiment and can be compared with its theoretically predicted values [19] for the triangular lattice [20], $\pi n_{e}\left\langle u^{2}\right\rangle_{T} \approx\left(1.6 T / e^{2} \chi^{-1} n_{e}^{1 / 2}\right) \ln \left(L / \lambda_{T}\right)$, where $\chi$ is the dielectric constant of the medium. The shorter length under the logarithm, $\lambda_{T}$, is the thermal magnetophonon wavelength, $T \sim 0.3 \nu\left(e^{2} \chi^{-1} n_{e}^{1 / 2}\right)\left(1 / \lambda_{T} n_{e}^{1 / 2}\right)^{3 / 2}$. The longer length $L$ is the correlation length of the crystal (related to the crystal pinning by charged acceptors, $L \geq n_{A}^{-1 / 2}$ ). At low temperatures, Eq. (2) can be expanded into the linear variation, $\tau_{m}(T) / \tau_{m}(0)-1 \approx$ $-\gamma \nu^{-2} \pi n_{e}\left\langle u^{2}\right\rangle_{T} \propto-T H^{2}$. The observed behavior shown in the inset to Fig. 3 is in a good agreement with such a linear temperature and square magnetic field dependence which is specific to the gapless magnetophonon excitation spectrum of a crystal. Finally, using Eq. (2) at $T=T_{c}$ we are able to find the value of the Lindemann parameter $\left\langle u^{2}\right\rangle_{T} n_{e} \approx(0.45 \pm 0.02) \times 10^{-2}$ for our system of electrons crystallized into a triangular lattice.

The authors thank A. MacDonald, P. Platzman, B. Shklovskii, and F. Stern for discussions. We also acknowledge the support from the Volkswagen Stiftung and (V.F.) from the Alexander von Humboldt Foundation.

[1] Yu. Losovik and V.I. Yudson, Pis'ma Zh. Eksp. Teor. Fiz. 22, 26 (1975) [JETP Lett. 22, 11 (1975)]; P.K. Lam and S.M. Girvin, Phys. Rev. B 30, 473 (1984); D. Levesque et al., ibid. 30, 1056 (1984).

[2] D.C. Tsui et al., Phys. Rev. Lett. 48, 1559 (1982).

[3] R.L. Willett et al., Phys. Rev. B 38, 7881 (1988).

[4] H.W. Jiang et al., Phys. Rev. Lett. 65, 633 (1990).

[5] V.J. Goldman et al., Phys. Rev. Lett. 65, 2189 (1990).

[6] E.Y. Andrei et al., Phys. Rev. Lett. 60, 2765 (1988).

[7] M.A. Paalanen et al., Phys. Rev. B 45, 13784 (1992).

[8] H. Buhmann et al., Phys. Rev. Lett. 66, 926 (1991); I.V. Kukushkin et al., Phys. Rev. B 45, 4532 (1992).

[9] E.M. Goldys et al., Phys. Rev. B 46, 7957 (1992).

[10] I.V. Kukushkin et al., Europhys. Lett. 23, 211 (1993).

[11] V.I. Fal'ko, Phys. Rev. B 49, 2242 (1994); 47, 3802 (1993).

[12] H. Fertig et al., Phys. Rev. Lett. 70, 1545 (1993); D. Liu et al., Phys. Rev. B 48, 11184 (1993).

[13] I.V. Kukushkin et al., Phys. Rev. B 40, 7788 (1989).

[14] At $\nu<1$ and low temperatures all the electrons and holes are fully polarized, and therefore we omit their spin indices.

[15] B. Janovici, Phys. Rev. Lett. 46, 386 (1981).

[16] K. Maki and X. Zotos, Phys. Rev. B 28, 4349 (1983).

[17] D.S. Fisher et al., Phys. Rev. B 20, 4692 (1979); E. Cockayne and V. Elser, ibid. 43, 623 (1991); I.M. Ruzin et al., ibid. 46, 3999 (1992).

[18] A.V. Chaplik, Zh. Eksp. Teor. Fiz. 62, 746 (1972) [Sov. Phys. JETP 35, 395 (1972)]; H. Fukuyama, Solid State Commun. 17, 1323 (1975).

[19] P.M. Platzman and H. Fukuyama, Phys. Rev. B 10, 3150 (1974); F.P. Ulinich and N.A. Usov, Zh. Eksp. Teor. Fiz. 76, 288 (1979) [Sov. Phys. JETP 49, 147 (1979)].

[20] L. Bonsall and A. Maradudin, Phys. Rev. B 15, 1959 (1977). 